\title{
State Space Versus SARIMA Modeling of the Nigeria's Crude Oil Export
}

\section{Omorogbe Joseph ASEMOTA}

Department of Statistics, University of Abuja, Nigeria.

Author's Email: asemotaomos@yahoo.com and omorogbe.asemota@uniabuja.edu.ng

Received: $7^{\text {th }}$ September 2015 / Revised: $20^{\text {th }}$ January $2016 /$ Accepted: $14^{\text {th }}$ August 2016 CIAppStat-SL2016

\begin{abstract}
This paper analyzes the Nigeria's crude oil export series using monthly data from January 1999 to December 2014. We employed the state space local level model with stochastic and deterministic seasonal to model the dynamic features in the Nigeria crude oil export. Our results clearly indicate that the local level model with deterministic seasonal is the most parsimonious model between the two state space models considered in this study. Also, a parsimonious SARIMA model is also fitted to the data. We compare the forecasting performance of the two parsimonious models and evaluate their forecasts using ex-post indicators such as mean absolute percentage error (MAPE), root mean square percentage error (RMSPE) and the Theil's U statistic. The forecast analysis and evaluation results indicate that the state space local level model with deterministic seasonal outperforms the Box-Jenkins model in shorter and medium - range forecasting horizons. Howbeit, the forecast of the SARIMA model improves in the longer horizon. The Theil's $U$ statistic also indicates that the state space local level model with deterministic seasonal and SARIMA model outperform the naïve model at most of the forecasting horizons. In conclusion, we recommend that the state space model with deterministic seasonal component should be used in shorter and medium range forecasting horizons of the Nigeria's monthly crude oil export. Howbeit, for longer forecasting horizon, ten months and above, the seasonal ARIMA model should be considered.
\end{abstract}

Key words: ARIMA, Crude oil, Filtering, Forecasting, Smoothing, State space model.

\section{Introduction}

The statistical modeling of time series started a long time ago with the introduction of the Autoregressive $(A R(p))$ and Moving Average (MA(q)) models by Yule (1927) and Slutsky (1927) respectively. Wold (1938) in his 
celebrated theorem "Wold's decomposition theorem" proved that any (stationary) time series can be decomposed into two different parts. The first part can be modeled as a linear combination of its own past (AR), and the second part is the MA component of a finite order. Box and Jenkins (1970; revised 1976) addressed the non-stationarity problem inherent in most economic time series by developing the ARIMA (p,d,q) model, where $d$ is the number of differencing employed to achieve stationarity. Doguwa and Alade (2015) found out that SARIMA model outperformed the SARIMA-X and autoregressive distributed lag model in longer forecasting horizon. They concluded that seasonality should be accounted for by using the SARIMA model. Etuk and Amadi (2013) also analyzed the monthly crude oil domestic production using the multiplicative SARIMA model. However, in recent years, there are growing concerns among time series researchers that Box-Jenkins models may not always lead to accurate forecasting especially in economic and business applications where the level of randomness is high and the constancy of patterns or parameters cannot be guaranteed, seeSalau (1998).

However, the state space method of time series analysis has a number of advantages over the Box-Jenkins models. State space method is more general and is based on the modeling of all the observed features of the data. The different features inherent in a series such as trend, seasonal, cycle, explanatory variables and interventions can be modeled separately before being put together in the state space model. The assumption of stationarity is not needed in state space model. The Box-Jenkins model however is based on the elimination of trend and seasonality by differencing until stationarity is attained. Durbin and Koopman (2001) noted that elimination of trend and seasonality by differencing may pose a problem in econometric applications where knowledge of these components are needed. For more discussions on the relative merit of state space models over the celebrated ARIMA models, see Durbin and Koopman (2001).

Harvey (1989) presented mathematical evidence of the equivalence relationships between the state space and the Box-Jenkins models. These equivalencies between the state space and Box-Jenkins models are also discussed in Harvey and Koopman (1992). Despite these theoretical equivalencies, the Box-Jenkins and state space approaches to time series modelling are quite different. For example, in Box-Jenkins approach, trend and seasonality are removed prior to modelling the series. In the state space framework, the different components are modelled explicitly in a structural framework in order to diagnose all the dynamics in the time series simultaneously.Although the literature on state space has increased recently, 
there is as yet scant empirical evidence on its performance relative to the more traditional Box-Jenkins methods.It is against this backdrop that this study is being proposed. The modeling is done using the Nigeria crude oil export from January 1999 to December 2014 as the sample period. Though, it is also a matter of empirical choice, we selected this sample period because Salau (1998) analyzed this series from January, 1987 to December 1994 using the innovation regression and the Box-Jenkins approach. Ayinde and Abdulwahab (2013) also analyzed the Nigerian crude oil export using seasonal autoregressive moving average (SARIMA) model using monthly data from January 2002 to December 2011. The rest of this paper is organized as follows. In section 2 , we discuss the modeling methodology employed in this work. The data and the empirical results are presented in chapter 3 . The performance of the fitted models is presented in section 4 . The final section concludes.

\section{Modeling Methodologies}

\subsection{State Space Approach: Local Level Model(LLM) with Seasonality}

The local level model or random walk-plus-noise model is a simple form of a linear Gaussian state space model for modelling series with no visible trend. The model contains only the level and irregular components; the single state (level) variable follows the random walk:

$$
\begin{array}{ll}
y_{t}=\mu_{t}+\varepsilon_{t}, & \varepsilon_{t} \sim \mathrm{N}\left(0, \sigma_{\varepsilon}^{2}\right) \\
\mu_{t+1}=\mu_{t}+\eta_{t}, & \eta_{t} \sim \mathrm{N}\left(0, \sigma_{\eta}^{2}\right)
\end{array}
$$

where $\varepsilon_{t}$ and $\eta_{t}$ are mutually uncorrelated white-noise processes with variance $\sigma_{\varepsilon}^{2}$ and $\sigma_{\eta}^{2}$. The interpretation of this model is that $\mu_{t}$ is an (unobservable) local level or mean for the process. The observable $y_{t}$ is the underlying process mean contaminated with the measurement error $\varepsilon_{\mathrm{t}}$. The aim of the analysis is to study the development of the state over time using the observed values, $y_{1}, \ldots, y_{n}$. However, when a time series consists of daily, monthly, or quarterly observations, the presence of seasonal effects should be investigated. In the state space framework, seasonality can be handled by building the seasonal effects directly into the model. Hence, adding seasonal components to equation (1) yields,

$$
\begin{array}{ll}
y_{t}=\mu_{t}+\gamma_{t}+\varepsilon_{t}, & \varepsilon_{t} \sim N\left(0, \sigma_{\varepsilon}^{2}\right) \\
\mu_{t+1}=\mu_{t}+\eta_{t}, & \eta_{t} \sim N\left(0, \sigma_{\eta}^{2}\right) \\
\gamma_{t+1}=-\sum_{j=1}^{s-1} \gamma_{t+1-j}+\omega_{t}, & \omega_{t} \sim N\left(0, \sigma_{\omega}^{2}\right)
\end{array}
$$


for $t=s-1, \ldots, n$. When the seasonal effect $\gamma_{t}$ is not allowed to change over time, we require $\omega_{t}=0$ for all $t=s-1, \ldots, n$. This is done by setting $\sigma_{\omega}^{2}=0$, and (2) is called the local level with deterministic seasonal model. When the seasonal effect $\gamma_{t}$ is allowed to vary over time, that is $\sigma_{\omega}^{2}>0$, the resulting model is called the local level with stochastic seasonal model. Since the crude oil export data consists of monthly observations, the periodicity of the seasonal is $s=12$. The stochastic formulation of the seasonal effect in (2) follows from the standard dummy variable methods of modelling seasonal pattern.

The Kalman filter is a statistical algorithm that enables certain computations to be carried out for a model cast in state space form. However, to obtain a more accurate estimate of the state vector, the smoothing algorithm is performed. Kalman smoothing provides us with a more accurate inference on $\mu_{t}$, since it uses more information than the filtering. Let $Y_{t-1}$ denote the set of past observations $\left\{y_{1}, \ldots, y_{t-1}\right\}$ and assuming the conditional distribution of $\mu_{t}$ given $Y_{t-1}$ is $\mathrm{N}\left(u_{t}, p_{t}\right)$ where $u_{t}$ and $p_{t}$ are to be determined. Assuming that $u_{t}$ and $p_{t}$ have been determined, the celebratedKalman filter equations for updating the above local level model from time $t$ to $t+1$ are given by:

$$
\begin{aligned}
& u_{t+1}=u_{t}+k_{t} v_{t}, \quad p_{t+1}=p_{t}\left(1-k_{t}\right)+\sigma_{\eta}^{2}, \quad k_{t}=p_{t} / f_{t}, \\
& v_{t}=\mathrm{y}_{t}-u_{t}, \quad f_{t}=p_{t}+\sigma_{\varepsilon}^{2},
\end{aligned}
$$

fort $=1,2, \ldots, \mathrm{n}$, where $v_{t}$ denotes the Kalman filter residual or prediction errors, $f_{t}$ is its variance and $k_{t}$ is the Kalman gain. A random walk like $\mu_{t}$ has no "natural" level and to handle the initial conditions $\left(u_{1}, p_{1}\right)$ for the nonstationary model, we employed the exact initial Kalman filter, (Durbin and Koopman, 2001). The Kalman smoothed state ( $\left.\hat{\mu}_{t}\right)$ and smoothed state variance $\left(V_{t}\right)$ can be calculated by the following backward recursions:

$\hat{\mu}_{t}=u_{t}+p_{t} r_{t-1}, \quad r_{t-1}=f_{t}^{-1} v_{t}+l_{t} r_{t}, \quad l_{t}=1-k_{t}=\sigma_{\varepsilon}^{2} / f_{t}, \quad t=n, \ldots, 1$ $V_{t}=p_{t}-p_{t}^{2} N_{t-1}, \quad N_{t-1}=f_{t}^{-1}+l_{t}^{2} N_{t}, \quad t=n, \ldots, 1 \quad$ (4) with $r_{n}$ and $N_{n}$ $=0$, for $t=n, \ldots, 1$. The unknown variance parameters in the state space model are estimated by the maximum likelihood estimation via the Kalman filter prediction error decomposition initialized with the exact initial Kalman filter.

Diagnostic checking in the state space models are based on the three assumptions concerning the residuals of the analysis. The residuals should 
satisfy these three properties, in order of importance; independence, homoscedasticity and normality. These assumptions are checked using the following test statistic;

The assumption of independence can be checked with theLjung-Box (1978) statistic defined as:

$\boldsymbol{Q}(\boldsymbol{k})=\boldsymbol{n}(\boldsymbol{n}+2) \sum_{l=1}^{k} \frac{r_{l}^{2}}{n-l} \sim \chi_{(k-w+1)}^{2}(5)$

for lags $l=1, \ldots, k ., r_{l}$ denotes the residual autocorrelation and $w$ is the number of hyperparameters (i.e. disturbance variances). The second most important assumption is the homoscedasticity of the residuals. This is checked using the following test statistic:

$\boldsymbol{H}(\boldsymbol{h})=\frac{\sum_{t=n-h+1}^{n} \varepsilon_{t}^{2}}{\sum_{t=d+1}^{d+h} \varepsilon_{t}^{2}} \sim \boldsymbol{F}(\boldsymbol{h}, \boldsymbol{h})$

where $d$ is the number of diffuse initial elements, and $h$ is the nearest integer to $(n-d) / 3$, and the assumption of normality is tested with:

$\boldsymbol{N}=\boldsymbol{n}\left(\frac{S^{2}}{6}+\frac{(K-3)^{2}}{24}\right) \quad \sim \chi_{2 d f}^{2}(7)$

where $\mathrm{S}$ denotes the skewness of the residuals, and $\mathrm{K}$ is the kurtosis.

\subsection{Multiplicative SARIMA $(p, d, q)(P, D, Q)_{S}$ Modeling Strategy}

The SARIMA model is an extension of ARIMA, where seasonality in the data is accommodated using seasonal difference. A multiplicative seasonal autoregressive integrated moving average model, SARIMA $(p, d, q)(P, D, Q)_{s}$ is given by;

$$
\Phi\left(L^{s}\right) \phi(L) \nabla_{s}^{D} \nabla^{d} y_{t}=\Theta\left(L^{s}\right) \theta(L) \varepsilon_{t}
$$

where $\phi(L)$ is the nonseasonal AR operator, $\theta(L)$ the nonseasonal MA operator, $\Phi\left(L^{s}\right)$ the seasonal AR operator, $\Theta\left(L^{s}\right)$ the seasonal MA operator, $L$ the lag operator, $\nabla^{d}$ the nonseasonaldthdifferencing, $\nabla_{s}^{D}$ the seasonal Dth differencing at s number of lags, s equals 12 for monthly data, $\mathrm{p}$ is the order of nonseasonal AR process, $\mathrm{P}$ is the order of seasonal AR process, $\mathrm{q}$ the order of nonseasonal MA process, and Q the order of seasonal MA process. Box-Jenkins (1976) determined the integer parameters $(\mathrm{p}, \mathrm{q})$ that govern the underlying process $\left\{y_{t}\right\}$ by examining the autocorrelations function (ACF) and partial autocorrelations (PACF) of the stationary series, $\left\{y_{t}\right\} \cdot{ }^{1}$ This step is highly

\footnotetext{
${ }^{1}$ For AR (p) models, the PACF has significant spikes through $\mathrm{p}$ and cut off after $\mathrm{p}$ while for MA (q), the ACF has significant spikes through q and for ARMA (p,q) both the ACF and PACF show different patterns (but all dampens after a while).
} 
subjective as different researchers using the same data set can arrived at different models. Hence, it is useful to entertain more than one structure for further analysis.

Although the state space local level and the ARIMA modeling strategies are different, however, there exists a link between the state space models and ARIMA model. In particular, the local level model is equivalent to an ARIMA $(0,1,1)$ model. This is demonstrated below;

using the specification of the local level model in equation (7),

$\nabla y_{t}=\mu_{t}-\mu_{t-1}+\varepsilon_{t}-\varepsilon_{t-1}=\eta_{t-1}+\varepsilon_{t}-\varepsilon_{t-1},(9)$

which take the form of a stationary $M A(1)$ process, taking the expectation of (9) yields

$E\left(\nabla y_{t}\right)=E\left(\eta_{t-1}\right)+E\left(\varepsilon_{t}\right)-E\left(\varepsilon_{t-1}\right)=0(10)$

The autocovariance function, $\gamma(k)=E\left(\nabla y \nabla y_{t-k}\right)$ of the process is given by,

$$
\begin{aligned}
& \gamma(0)=E\left[\left(\nabla y_{t}\right)^{2}\right]=E\left[\left(\eta_{t-1}+\varepsilon_{t}-\varepsilon_{t-1}\right)^{2}\right]=E\left[\eta_{t-1}^{2}+\varepsilon_{t}^{2}+\varepsilon_{t}^{2}\right]=\sigma_{\eta}^{2}+2 \sigma_{\varepsilon}^{2} \\
& \gamma(1)=E\left[\left(\eta_{t-1}+\varepsilon_{t}-\varepsilon_{t-1}\right)\left(\eta_{t-2}+\varepsilon_{t-1}-\varepsilon_{t-2}\right)\right]=-E\left(\varepsilon_{t-1}^{2}\right)=-\sigma_{\varepsilon}^{2} \\
& \gamma(k)=0, \quad k \geq 2
\end{aligned}
$$

Hence, the autocorrelation function of $\nabla y_{t}$ is given by

$$
\rho_{k}=\frac{\gamma(k)}{\gamma(0)}= \begin{cases}-\sigma_{\varepsilon}^{2} /\left(\sigma_{\eta}^{2}+2 \sigma_{\varepsilon}^{2}\right), & k=1(12) \\ 0 \quad, & k \geq 2\end{cases}
$$

The autocorrelation function (ACF) represented in (12) is the same as the ACF of the $M A(1)$ process. This implies that the local level model is equivalent to an ARIMA $(0,1,1)$ model. Harvey (1989) presented a summary of more equivalencies between the state space and ARIMA models. Also, it can be demonstrated that the ARIMA models can be represented in the state space framework and fitted by the state space methods. For a comprehensive demonstration of these representations, see Hamilton (1994, pg. 374-377).

\section{Data and Empirical Results}

\subsection{The Data}

The monthly data of Nigeria's crude oil export from January, 1999 to December 2014 are examined in this study. Specifically, the data from January 1999December 2013 is used in the estimation of the models; while data from January 2014 - December 2014 is used to evaluate the forecasting performance of the models. The monthly crude oil export data, measured in millions barrels were 
obtained from the Nigeria National Petroleum Corporation (NNPC) website. The plots of the series and log-transformed data are presented in Figure 1 and 2 respectively.

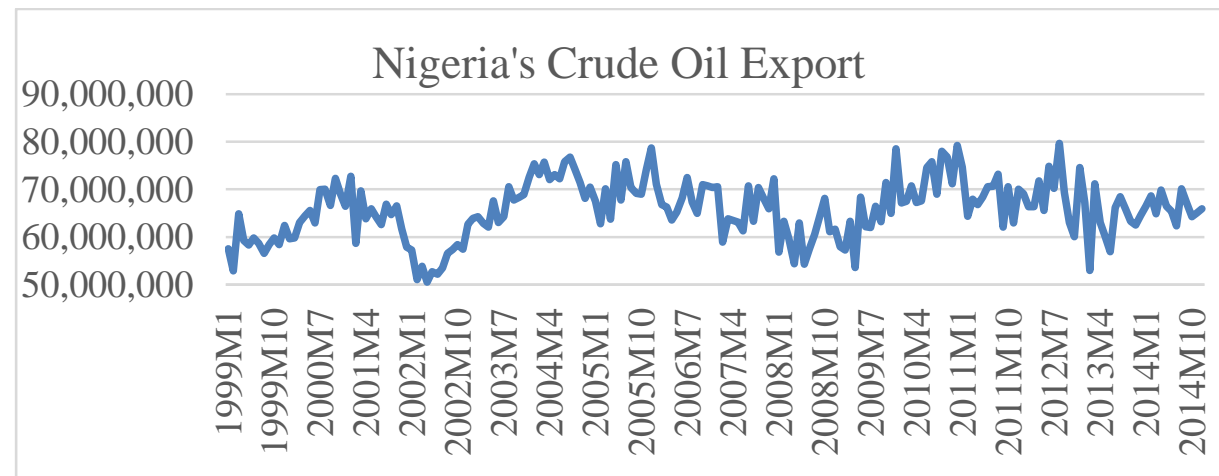

Fig.1 Time Series Plot of Nigeria's Monthly Crude Oil Export

Examination of plots of both the original data and the log-transformed data revealed that nonstationary features are clearly inherent in both series. The crude oil export series fluctuates but generally increased until it fell to its lowest level in April, 2002 and again gradually rose to unimaginable level in December, 2010 and August, 2012, this may be due to the amnesty program introduced by the Federal Government of Nigeria in 2009. Hence, any statistical model used to model this series should take these non-stationary features into consideration.

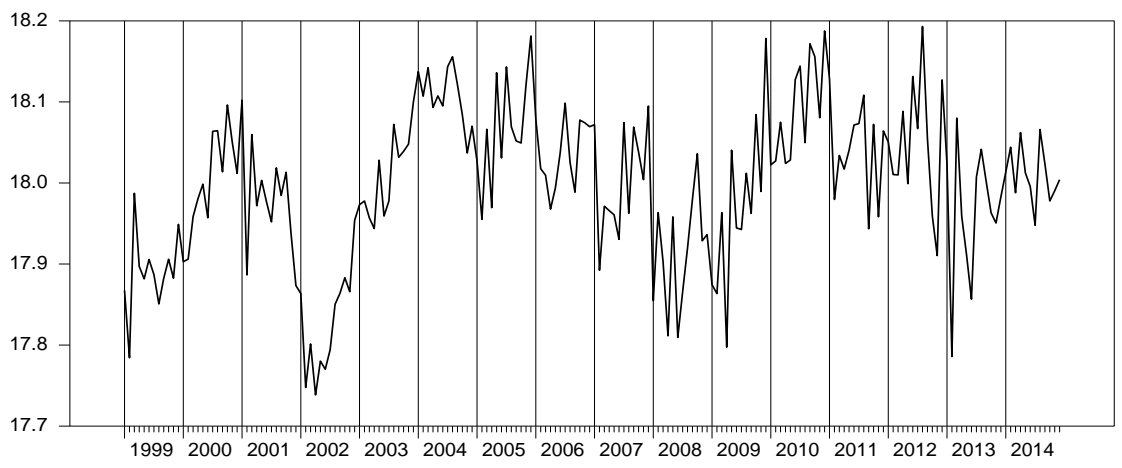

Fig.2Time Series Plot for the Log of Nigeria's Monthly Crude Oil Export

After taking log, the variance of the series is relatively stable and very small (0.009). Figure 2 indicates that for most of the years considered in this work, more crude oil is exported towards the end of the year than in other periods of a year. This recurring pattern is an indication of a seasonal effect. 


\subsection{State Space Model Estimation Results.}

Since seasonalitymay be present in a monthly time series data, we employed the LLMwith stochastic seasonal and LLM with deterministic seasonalmodels described in section 2.1 to the $\log$ transformed series. First, using the data, we estimate the unknown variance parameters (hyperparameters) of the models using maximum likelihood method. This is maximized using the BFGS (Broyden-Fletcher-Goldfarb-Shannon) optimization method because the BFGS approximates the Newton's method and has proven to have good performance even for non-smooth optimizations, see Lewis and Overton (2008).

The estimation results for the local level model with stochastic seasonal is presented in Table 1.

Table 1: Estimation Results for LLM with Stochastic Seasonal (1999:1 - 2013:12)

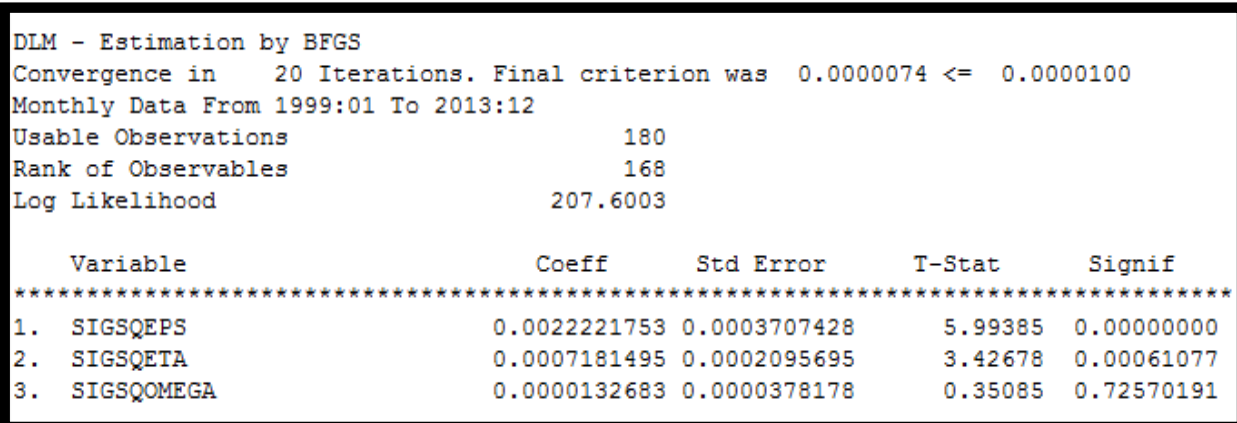

Note: SIGSQEPS, SIGSQETA and SIGSQOMEGA denote estimates of $\sigma_{\varepsilon}^{2}, \sigma_{\eta}^{2}$ and $\sigma_{\omega}^{2}$ respectively

The estimation results in Table 1 indicate that the estimated hyper-parameters (disturbance variances) of the measurement and level equations are highly statistically significant; however, the estimated hyperparameter for the seasonal component is not statistically significant. This indicates that any seasonal pattern in the observed time series hardly changes over the years, which is confirmed by inspection of the plot of the smoothed seasonal effect in Figure 4. Also, there are 180 observations in our series; however, the estimation is done using only the final 168 observation. This is because 12 diffuse initial state values are estimated (11 for the seasonal components and 1 for the local level components).

In Figure 3, the dynamic evolution of the level, seasonal components and the fitted values obtained by the Kalman filter are presented graphically together with their variances and the prediction errors (residuals). The evolution of Nigeria crude oil export is reflected by the estimated level component and is presented in the upper graph of Figure 3. The plot indicates that the periods of 
highest level of crude oil export occurred in the 2010s, while the lowest level of export occurred in the 2002s. The highest level witnessed in the 2010s may have resulted from the benefits of the Amnesty program launched by the Federal Government of Nigeria in June, 2009. One interesting obvious features of the graph is that, the Kalman filtered state variances converges to a constant values as the sample size increases which empirically confirms that the local level model has a steady state solution, see (Durbin and Koopman, 2001, page 32).
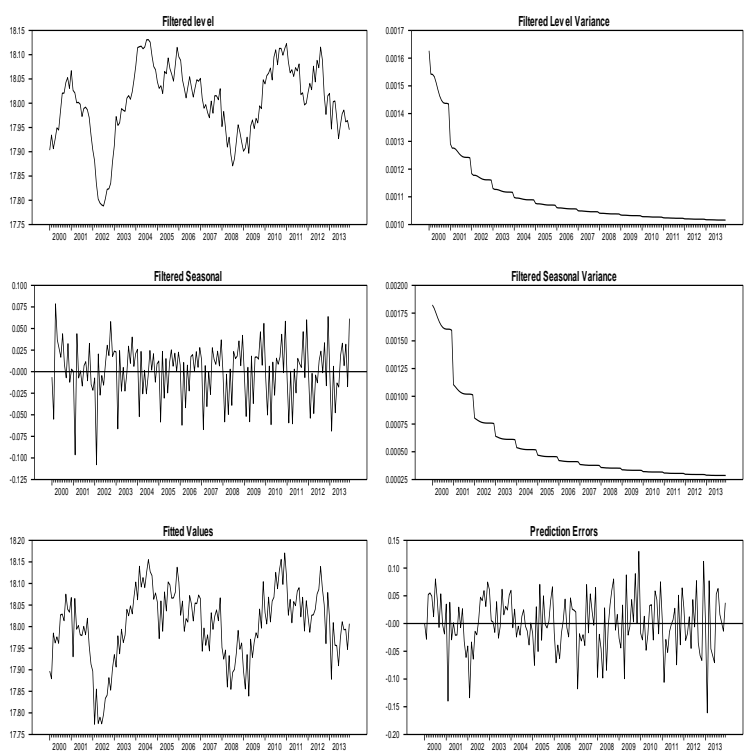

Fig.3.Kalman Filter Output for Local level+Stochastic Seasonal

Fig. 3:Kalman Filter Output for LLM and Stochastic Seasonal

Figure 4 presents the output of the Kalman smoothing recursion estimates of the level, seasonal and the fitted values of the series together with the state variances of the level and seasonal component, and the smoothed prediction errors. Comparing the graphs of the Kalman filtered level and the smoothed level in figures 3 and 4; we see that the graph in figure 4 is smoother than that of Figure 3. Also, from the graph of the smoothed seasonal pattern in figure 4, it is very obvious that the seasonal pattern in the crude oil export has been relatively constant over the years. The plot of the correlogram in Figure 5 indicates that the null hypothesis of independence of the residuals is not rejected, an indication that the residuals are close to independent random process (white noise). This is confirmed by the inspection of the diagnostics of the model presented in Table 2. The diagnostic tests for independence, homoscedasticity, and normality of the residuals are all satisfactory. However, 
since the variance of the seasonal disturbances in local level model with stochastic seasonal is found to be very small and not statistically significant (see Table 1), we presents the results of analysis of the Nigeria crude oil export time series with a local level model and a deterministic seasonal.
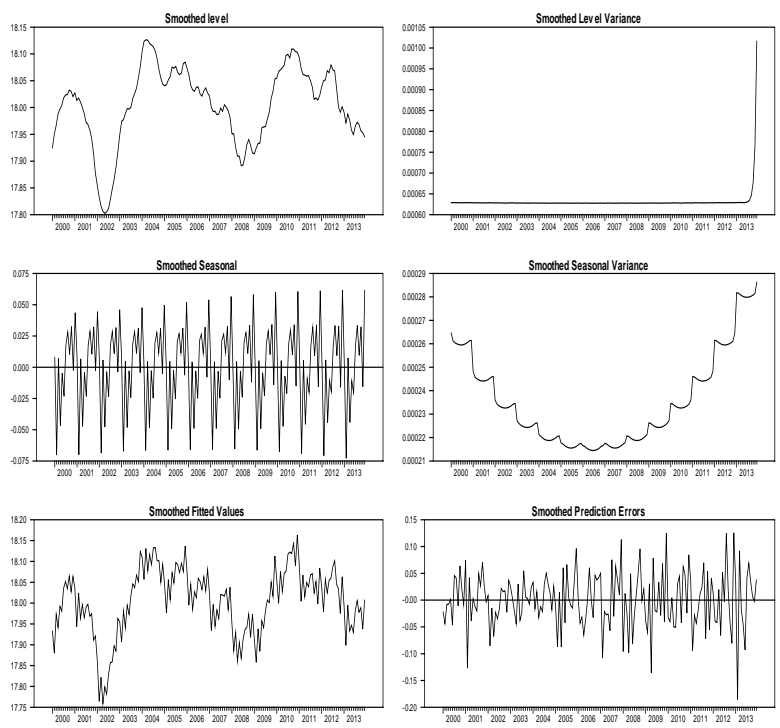

Fig.4.Kalman Smoothed Output for Local level+Stochastic Seasonal

Fig 4:Kalman Smoothed Output for LLM with Stochastic Seasonal

Table 2: Diagnostics Results for Local Level Model with Stochastic Seasonal

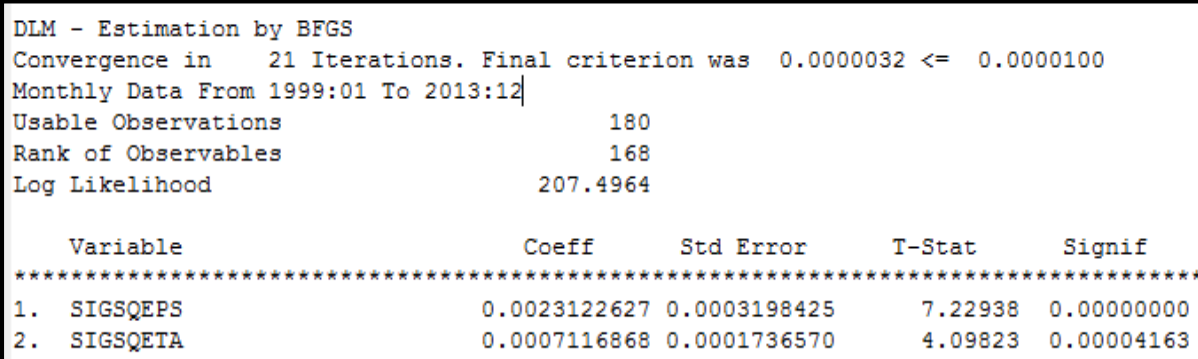

Note: SIGSQEPS and SIGSQETA denote estimates of $\sigma_{\varepsilon}^{2}$ and $\sigma_{\eta}^{2}$ respectively 


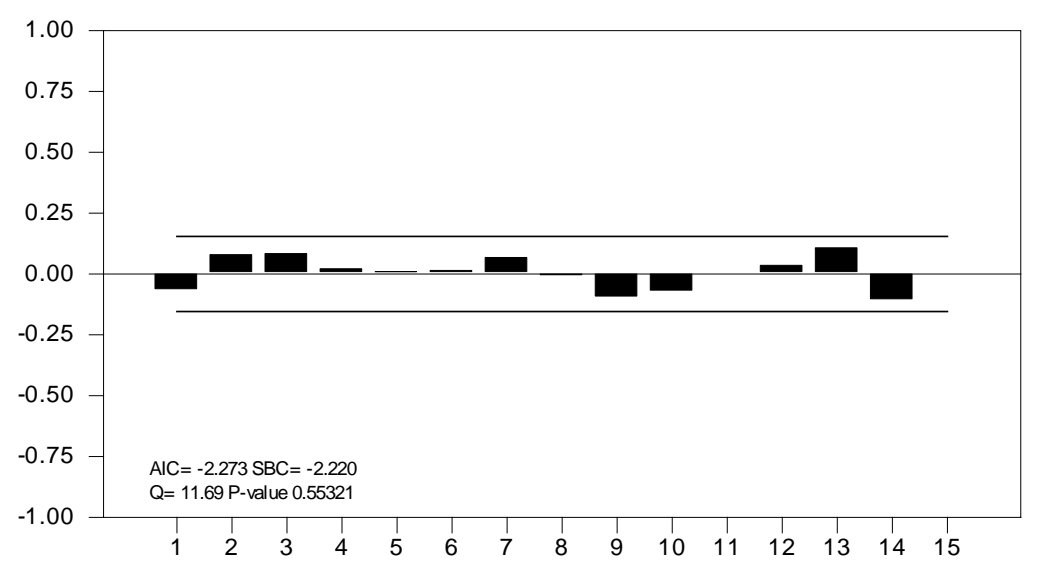

Fig.5:Correlogram for the Local Level Model with Stochastic Seasonal

Table 3: Estimation Results for Local LLM with Deterministic Seasonal

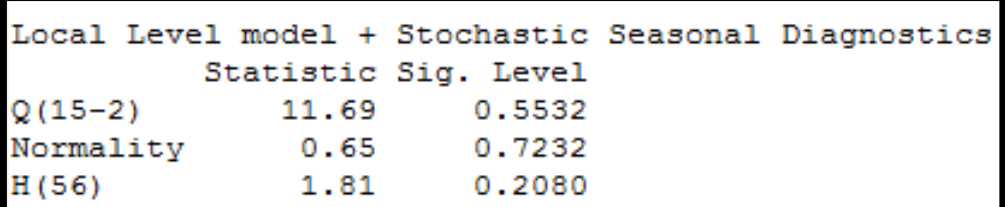

The estimation results display above shows that the hyper-parameters are both highly statistically significant. Note that, the variance of the seasonal disturbances $\sigma_{\omega}^{2}$ is fixed at zero in this estimation.Weperformed the Kalman filtering and smoothing based on these two estimates. We present the results of the Kalman filter and Kalman smoothed estimates of the local level model with deterministic seasonal in figure 6 and figure 7 respectively Since the output is very similar to the output of the local level model with stochastic seasonal (as a result of the small and insignificantvariance), the discussion of the results is similar to the previous discussion. However, the constant seasonal pattern in the series is clearly revealed in Figure 7. The results of the diagnostic tests for the residuals of the local level model with deterministic seasonal are again very satisfactory. 

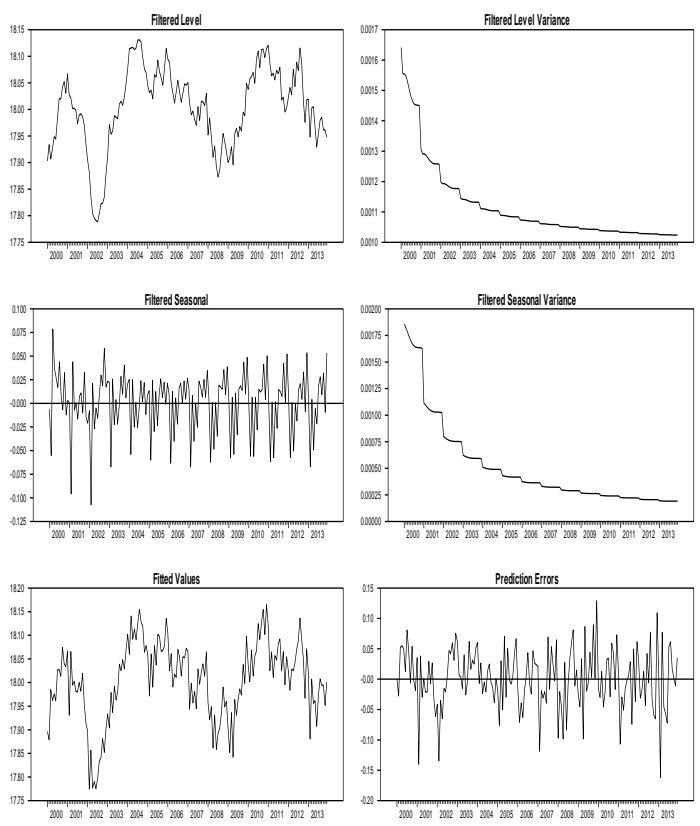

Fig.6.Kalman Filter Output for Local level + Determinisicic Seasonal

Fig 6:Kalman Filter Output for LLM and Deterministic Seasonal
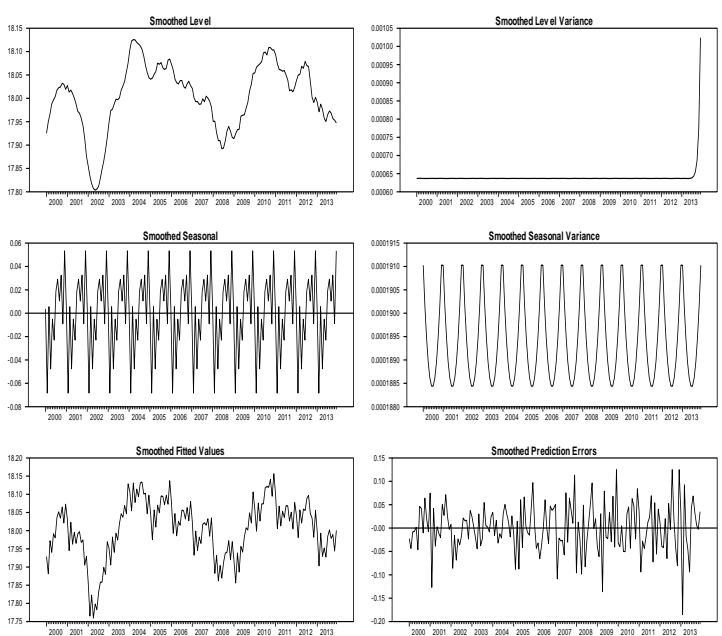

Fig.7.Kalman Smoothed Output for Local level + Deterministic Seasonal

Fig 7:Kalman Smoothed Output for LLM and Deterministic Seasonal 
In order to choose the most appropriate model between the two competing state space models, the Akaike Information Criterion (AIC) and the Schwarz's Bayesian Criterion (SBC) of the two models are examined, see Commandeur and Koopman (2007). The AIC and SBC impose a penalty for inclusion of additional parameters in the models. In the information criterion approach, models that yield smaller values for the criterion are preferred, and regarded as best fitting model. From figure 5, the AIC and SBC for the local level model with stochastic seasonal are -2.273 and -2.220 respectively, while the AIC and SBC for the local level model with deterministic seasonal displayed in figure 8 are -2.283 and -2.448 respectively. Thus, the fit of the local level model with deterministic seasonal is slightly better than the fit of the model with stochastic seasonal component. Since the log-likelihood values of the two models are almost identical, 207.60 and 207.50 for the local level model with stochastic seasonal and the local level model with deterministic seasonal respectively, the improved fit of the second model can be completely attributed to its greater parsimony.Commandeur and Koopman (2007) pointed out that, in state space modelling, a small and insignificant state disturbance variance indicates that the corresponding state component may as well be treated as a deterministic effect, resulting in a more parsimonious model.

Table 4: Diagnostics for Local Level Model with Deterministic Seasonal

Local Level model + Deterministic Seasonal Diagnostics

Statistic Sig. Level

Q(15-1) $\quad 11.26 \quad 0.6653$

Normality $\quad 0.40 \quad 0.8203$

$\begin{array}{lll}\mathrm{H}(56) & 1.83 & 0.2058\end{array}$ 


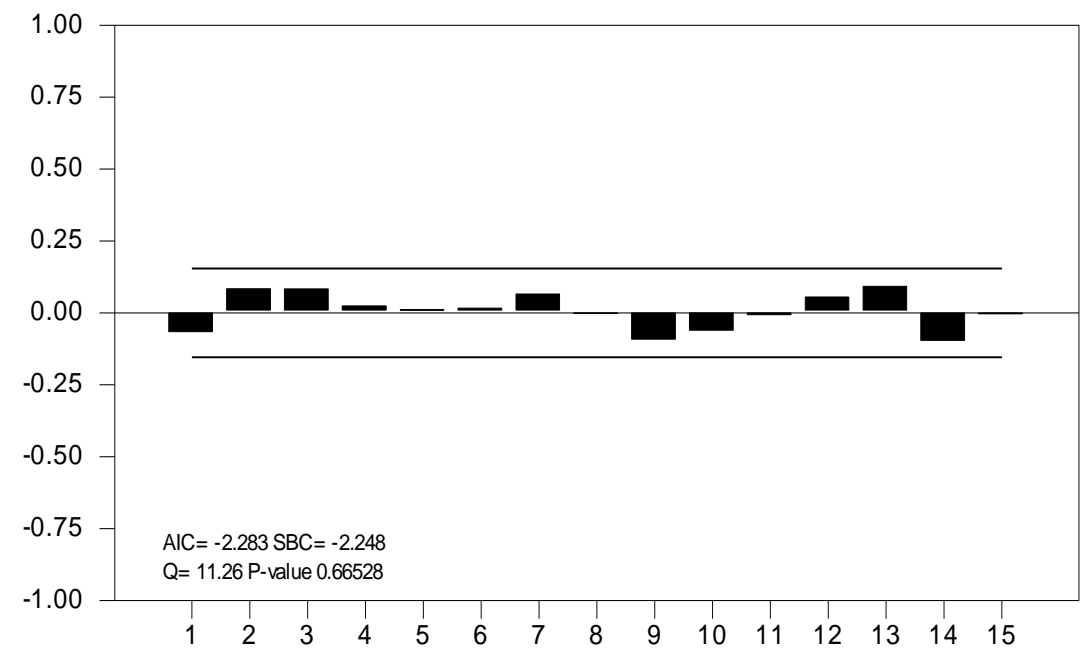

Fig. 8:Correlogram for the Local Level Model with Deterministic Seasonal

\subsection{SARIMA Model Estimation Results}

In this framework, model building commences with the examination of the plots of the log-differenced and seasonally adjusted series and the autocorrelation presented in Figures 9 and 10 respectively.

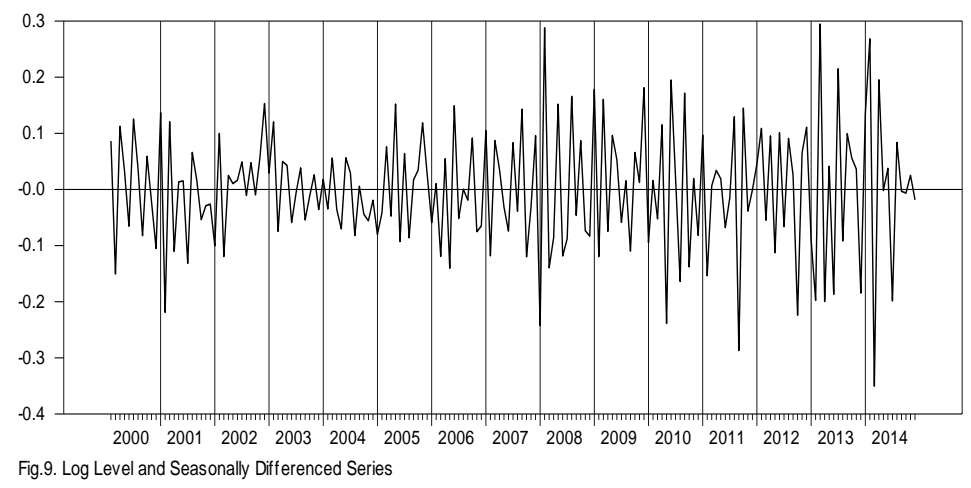

Fig 9: Log Level and Seasonally Differenced Series 
Figure 9 indicates that stationarity has been induced in the seasonally adjusted series. This is further confirmed by the correlogram displayed in Figure 10. We used the behaviour of the autocorrelations and partial autocorrelations displayed in figure 10 to identify some candidate models which are further subjected to model selection analysis to obtain the most parsimonious SARIMA model for the data.

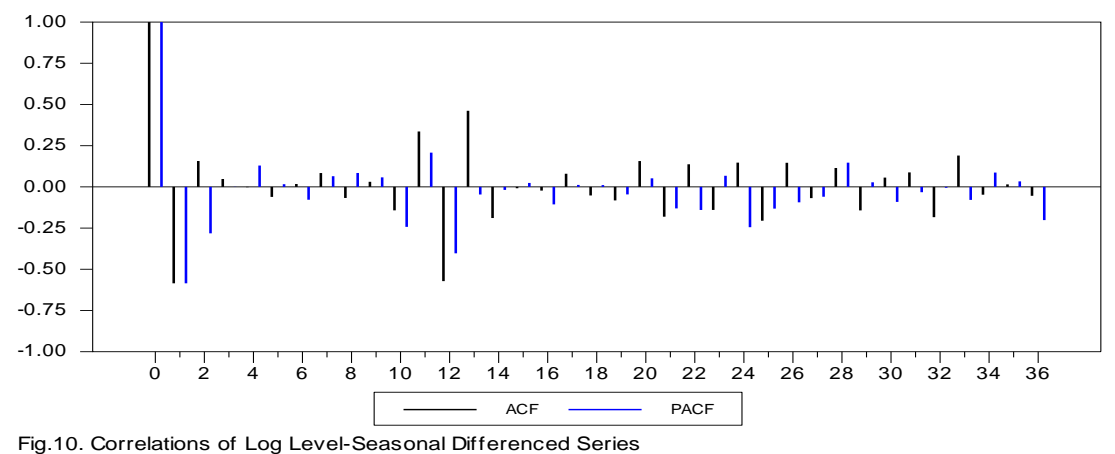

Fig. 10: Correlations of Log Level-Seasonal Differenced Series

From a close observation of the plots of ACF and PACF, we noticed that both the ACF and PACF displayed significant spikes at lag 1 and 12 with ACF and PACF values for lag 1 and $12(-0.5852,-0.58521)$ and $(-0.5719,-0.40316)$ respectively.Based on these patterns, some possible candidate SARIMA models are identified. We proceed to estimation and further statistical analysis with the four possible models. We summarized the results in Table 5.From Table 5, SARIMA structures 3 and 4 seem to be the most competitive models. Their parameters estimates are all significant and both have the minimum standard error of estimate.

The most important summary statistics for measure of goodness of fit are the likelihood function (for maximum likelihood estimation), and the Q-test statistic for independence of the residuals. For a well fitted model, the Q-test statistic is expected to be statistically insignificant. In addition, another criteria for comparing competing models are the Akaike information criteria (AIC) and the Schwarz's Bayesian criteria (SBC). When considering several ARMA models, we choose the one with the lowest AIC and SBC. Based on these important statistics, ARIMA structure 3, seems to provide the best satisfactory fit to the logged crude oil export series. This model has the highest likelihood function,smallest standard error of estimate and the minimum AIC and BIC among all the ARIMA structures considered. Furthermore, the Q-test statistic indicates that the residuals do not suffer from autocorrelation. We will now compare the forecasting performance of the two modeling strategies considered in this work using the crude oil export series. 
Table 5: SARIMA MODELS Results

\begin{tabular}{|c|c|c|c|c|c|}
\hline $\begin{array}{l}\text { SARIMA } \\
\text { Structures }\end{array}$ & Parameter Estimates & P-value & $\begin{array}{l}\text { Likelihood\& } \\
\text { AIC }\end{array}$ & $\begin{array}{l}\text { Standard } \\
\text { Error of } \\
\text { Estimate }\end{array}$ & Q-Statistics \\
\hline 1. & $\begin{array}{l}\operatorname{AR}\{2\}=0.1610 \\
\operatorname{MA}\{1\}=-0.5755 \\
\operatorname{SAR}\{12\}=-0.4410\end{array}$ & $\begin{array}{l}0.0672 \pm \\
0.0000 \ddagger \\
0.0000 \ddagger\end{array}$ & $\begin{array}{l}192.8788 \\
\mathrm{AIC}=-2.274 \\
\mathrm{SBC}=-2.218\end{array}$ & 0.0763 & $\begin{array}{l}47.76 \\
(0.047+)\end{array}$ \\
\hline 2. & $\begin{array}{l}\operatorname{AR}\{1\}=-0.6357 \\
\operatorname{AR}\{2\}=-0.2179 \\
\operatorname{SAR}\{12\}=-0.4279\end{array}$ & $\begin{array}{l}0.0000 \ddagger \\
0.0049 \pm \\
0.0000 \ddagger\end{array}$ & $\begin{array}{c}194.8223 \\
\mathrm{AIC}=-2.427 \\
\mathrm{SBC}=-2.364\end{array}$ & 0.0754 & $\begin{array}{l}44.01 \\
(0.095 \ddagger)\end{array}$ \\
\hline 3. & $\begin{array}{l}\operatorname{AR}\{1\}=-0.6462 \\
\operatorname{AR}\{2\}=-0.2565 \\
\operatorname{MA}\{12\}=-0.9247 \\
\operatorname{SAR}\{24\}=-0.0245\end{array}$ & $\begin{array}{l}0.0000 \ddagger \\
0.0004 t \\
0.0000 \ddagger \\
0.8090\end{array}$ & $\begin{array}{l}208.8973 \# \\
\mathrm{AIC}=-2.454 * \\
\mathrm{SBC}=- \\
2.379 *\end{array}$ & $0.0655^{*}$ & $\begin{array}{l}28.70 \\
(0.635)\end{array}$ \\
\hline 4. & $\begin{array}{l}\operatorname{AR}\{1\}=-0.5974 \\
\operatorname{AR}\{2\}=-0.1941 \\
\operatorname{AR}\{12\}=-0.3696 \\
\operatorname{SAR}\{12\}=-0.2087\end{array}$ & $\begin{array}{l}0.0000 t \\
0.0060 \dagger \\
0.0000 t \\
0.0044 t\end{array}$ & $\begin{array}{l}203.4190 \\
\mathrm{AIC}=-2.388 \\
\mathrm{SBC}=-2.314\end{array}$ & 0.0713 & $\begin{array}{l}34.61 \\
(0.344)\end{array}$ \\
\hline
\end{tabular}

NOTES: $\$, \pm$ and $\dagger$ denote significant at the $1 \%, 5 \%$ and $10 \%$ levels respectively. *denotes the minimum AIC and BIC \# denotes the maximum likelihood and $* *$ denotes the minimum standard error of estimate.

The chosen seasonal ARIMA is of the form:

$$
\left(1-\emptyset_{1} B-\emptyset_{2} B^{2}\right)\left(1-\phi_{2} B^{24}\right) y_{t}=\left(1+\Theta_{12} B^{12}\right) \varepsilon_{t}(13)
$$

Using the properties of the backshift operator B, (19) can be expressed as:

$\Delta^{12} \operatorname{dlog}\left(o i l_{t}\right)=\emptyset_{1} y_{t-1}-\emptyset_{1} \phi_{2} y_{t-25}+\emptyset_{2} y_{t-2}+\phi_{2} y_{t-24}-\emptyset_{2} \phi_{2} y_{t-26}+$ $\Theta_{12} \varepsilon_{t-12}+\varepsilon_{t}$

where $\varepsilon_{t}$ is a white noise, $y_{t}$ is the $\log$ crude oil data, $\emptyset_{1}, \emptyset_{2}, \phi_{2}$ and $\Theta_{2}$ are presented in Table 5.

\subsection{Forecasting with the Fitted Models}

In this section, we assess the forecasting performance of state space model relative to Box-Jenkins models. The state space forecasting technique via Kalman filtering is extremely flexible and can deal with all types of data patterns and changes in patterns. The traditional Box-Jenkins approach is also general and can handle effectively many series encounter in reality. Besides, previous research has demonstrated that the Box-Jenkins forecast outperforms the Holt-Winters and stepwise autoregression forecasts, (Newbold and Granger, 1974). In addition, 
Forecasts based on the state space (local level) model may be readily obtained based on the Kalman filter procedure. In this approach, forecasting is done by filtering the observations $y_{1}, \ldots, y_{T}, y_{T+1}, \ldots, y_{T+L}$ using the recursion equation (4) stated in section 2.1 and treating the last $l$ observations $y_{T+1}, \ldots, y_{T+L}$ as missing. If we denote the minimum mean square error (MMSE) forecast of $y_{T+l}$ by $\hat{y}_{T+l}$ given the time series $y_{1}, \ldots, y_{T}$ for $l=1,2, \ldots$ ., $L$, using the local level model, the MMSE forecast $\hat{y}_{T+l}$ and its variance $\tilde{f}_{T+l}$ are given by:

$\hat{y}_{T+l}=E\left(y_{T+l} / Y_{T}\right)=E\left(\mu_{T+l} / Y_{T}\right)+E\left(\varepsilon_{T+l} / Y_{T}\right)=\hat{\mu}_{T+l}$ $\tilde{f}_{T+l}=\operatorname{Var}\left(y_{T+l} / Y_{T}\right)=\operatorname{Var}\left(\mu_{T+l} / Y_{T}\right)+\operatorname{Var}\left(\varepsilon_{T+l} / Y_{T}\right)=\tilde{p}_{T+l}+\sigma_{\varepsilon}^{2}$

for $l=1,2, \ldots, L$. Comparing equations (15) and (4) implies that the forecasts and their error variances are delivered by applying the Kalman filtering in the standard way with $v_{t}=0$ and $k_{t}=0$ throughout the forecast horizon. ${ }^{2} \mathrm{On}$ the other hand, forecast for ARIMA model can be computed directly from the ARIMA model equation by replacing: (1) future values of the error term by zero (2) future values of the $y_{t}$ by their conditional expectation, and (3) present and past values of $y_{t}$ and $\varepsilon_{t}$ by their observed values.

\subsection{Forecasting performance}

According to Hendry and Clements (2003), a forecast is any statement about the future. The state space and the SARIMA models are estimated based on the monthly data from 1999:1- 2013:12, and ex post forecasts are generated for the time period 2014:1 -2014:12. The plot of the actual crude oil values for 2014:1 - 2014:12 versus the SARIMA and state space models forecasts are depicted in Figure 11. The forecast analysis clearly shows that the state space model forecasts clearly outperform the Box-Jenkins model at all forecasting horizons.Various error measures are available for forecasts evaluation; however, we limit our forecast evaluation to three criteria. These are the mean absolute percentage error (MAPE), root mean square percentage error (RMSPE) and the Theils U statistic. According to Lewis (1982), the MAPE is the most useful measure in comparing the accuracy of forecasts between different methods since it measures relative performance. In addition, Makridakis and Winkler (1983) also recommended the use of MAPE when evaluating forecasts involving

\footnotetext{
${ }^{2}$ This brief discussion is extracted from Durbin and Koopman (2001), for more illuminating discussion see the Durbin and Koopman (2001).
} 
different series. If the analyst thinks the loss function is quadratic, rather than linear, the RMSPE comes in handy.The RMSPE generates forecasts in the same unit as the measured variable and so is a better descriptive statistics (Chatfield 2000). For detailed explanation why the MAPE and RMSPE are good measures for forecasts evaluation, see Witt and Witt (1992).

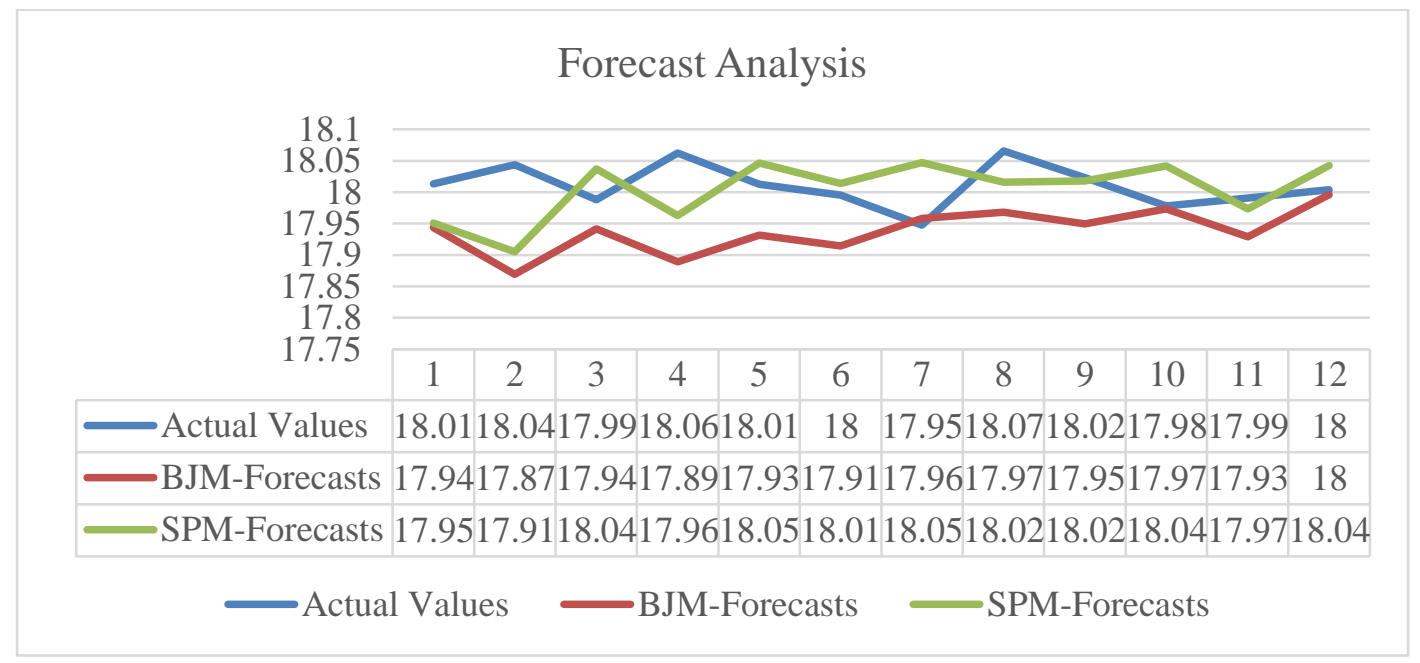

Fig 11:Plots of Actual values versus Box-Jenkins and State Space Models Forecasts

Assuming $n$-period steps ahead forecasts are to be generated, these two criteria are illustrated as:

$$
\begin{aligned}
& \text { MAPE }=\frac{1}{n} \sum_{t=1}^{n} \frac{\left|e_{t}\right|}{y_{t}} \\
& \text { RMSPE }=\sqrt{\frac{1}{n} \sum_{t=1}^{n}\left[\frac{e_{t}}{y_{t}}\right]^{2}}
\end{aligned}
$$

In addition to these two criteria, the Theil's $U$ statistic is also used in comparing forecast accuracy of different models. It has the advantage of providing an immediate comparison of the forecasts with those of the naive method. The Theil's U statistic is given by:

$$
U_{t}=\frac{\sqrt{\sum_{t=1}^{n}\left(A_{t}-F_{t}\right)^{2}}}{\sqrt{\sum_{t=1}^{n}\left(A_{t}-A_{t-1}\right)^{2}}}
$$


State Space Versus SARIMA Modeling Strategies

where $A_{t}$ is the actual value in time $t, F_{t}$ is the forecast value in time $t$, and the denominator is the RMSE of the naïve model forecast. ${ }^{3}$

Table 6: Forecasting performance -MAPE, RMSPE andTheil's Statistics

\begin{tabular}{|c|c|c|c|c|}
\hline $\begin{array}{l}\text { Forecast } \\
\text { horizon }\end{array}$ & $\begin{array}{l}\text { Forecasting } \\
\text { Model }\end{array}$ & MAPE & RMSPE & Theil's U \\
\hline \multirow[t]{2}{*}{1} & SPM & $0.0622(1)$ & $0.0622(1)$ & $2.0270(1)$ \\
\hline & BJM & $0.0697(2)$ & 0.0697 (2) & $2.2701(2)$ \\
\hline \multirow[t]{2}{*}{2} & SPM & 0.0929 (1) & 0.0929 (1) & $1.0305(1)$ \\
\hline & BJM & $0.1222(2)$ & $0.1330(2)$ & $1.3380(2)$ \\
\hline \multirow[t]{2}{*}{3} & SPM & $0.0020(1)$ & $0.0020(1)$ & $0.6554(1)$ \\
\hline & BJM & $0.0045(2)$ & $0.0054(2)$ & $1.6945(2)$ \\
\hline \multirow[t]{2}{*}{4} & SPM & 0.0039 (1) & $0.0045(1)$ & $0.7374(1)$ \\
\hline & BJM & $0.0064(2)$ & $0.0072(2)$ & $1.5322(2)$ \\
\hline \multirow[t]{2}{*}{5} & SPM & $0.0034(1)$ & $0.0034(1)$ & $0.2300(1)$ \\
\hline & BJM & $0.0060(2)$ & $0.0067(2)$ & $0.3855(2)$ \\
\hline \multirow[t]{2}{*}{6} & SPM & $0.0025(1)$ & $0.0025(1)$ & $0.1465(1)$ \\
\hline & BJM & $0.0058(2)$ & $0.0064(2)$ & $0.4654(2)$ \\
\hline \multirow[t]{2}{*}{7} & SPM & $0.0053(2)$ & $0.0071(2)$ & 0.0641 (2) \\
\hline & BJM & $0.0050(1)$ & $0.0060(1)$ & $0.0572(1)$ \\
\hline \multirow[t]{2}{*}{8} & SPM & $0.0064(1)$ & 0.0064 (1) & 0.9717 (1) \\
\hline & BJM & $0.0071(2)$ & $0.0066(2)$ & $1.7501(2)$ \\
\hline \multirow[t]{2}{*}{9} & SPM & $0.0040(1)$ & $0.0040(1)$ & 0.9869 (1) \\
\hline & BJM & $0.0050(2)$ & $0.0057(2)$ & $1.5435(2)$ \\
\hline \multirow[t]{2}{*}{10} & SPM & 0.0048 (2) & $0.0083(2)$ & $0.9853(2)$ \\
\hline & BJM & $0.0045(1)$ & $0.0054(1)$ & 0.6985 (1) \\
\hline \multirow[t]{2}{*}{11} & SPM & 0.0029 (1) & 0.0029 (1) & $0.6011(1)$ \\
\hline & BJM & $0.0044(2)$ & $0.0053(2)$ & $0.7223(2)$ \\
\hline \multirow[t]{2}{*}{12} & SPM & $0.0031(2)$ & $0.0038(2)$ & $0.2790(2)$ \\
\hline & BJM & $0.0024(1)$ & $0.0032(1)$ & 0.1701 (1) \\
\hline
\end{tabular}

Note: Values in parenthesis are ranks. The Theil's U statistic is a ratio of the RMSE for the model to the RMSE of a "naïve model". A value in excess of one is not promising since it implies the model did worse than the naïve model.

\footnotetext{
${ }^{3}$ A Naïve method simply states that the future forecasts are equal to the latest available value $y_{t}$, where $t$ is the most recent time period. Hence, the forecast is a horizontal line extrapolation.
} 
The results of these criteria are presented in Table 6. The forecasting ability of the state space model is examined with respect to the best fitting SARIMA model and the nä̈ve model is used as a bench mark for comparing the forecasting ability of the models.From the forecasts evaluation results presented in Table 6, the MAPE and RMSPE indicate that the state space local level model with deterministic seasonal outperforms the Box-Jenkins model in shorter and medium - range forecasting horizons. Howbeit, the forecast of the SARIMA model improves in the longer horizon. Our results show that the SARIMA model outperforms the state space model in forecasting horizons 10 and 12. The Theil's U statistic also indicates that the state space local level model with deterministic seasonal and SARIMA model outperform the naïve model at most of the forecasting horizons. Since the Theil's $U$ is less than one in most of the forecasting horizons. Hence, the SARIMA model remains a powerful modelling and forecasting technique for univariate time series data.

\section{Conclusions}

This paper fits a suitable state space and Box-Jenkins models to Nigeria's crude oil export and compared the forecasting performance of the fitted models. Prior to the forecasting competition, we employed two state space models, the local level model with stochastic and deterministic seasonal to model the dynamic features in the Nigeria crude oil export. The Kalman filter and smoothed estimates of the two models are quite identical and both models passed the diagnostic tests for the residuals. However, the AIC and SBC criteria clearly indicate that the fit of the local level model with deterministic seasonal is better than the model with stochastic seasonal component.In addition, an appropriate SARIMA model is also fit to the logged crude oil export series. The forecast evaluation of the most parsimonious state space and SARIMA models is carried out using the MAPE, RMSPE and the Theil's U criteria. Employing the MAPE and RMSPE criteria as the forecast accuracy measures, the results indicate that the state space local level model with deterministic seasonal outperforms the Box-Jenkins model in shorter and medium - range forecasting horizons. Howbeit, the forecast of the SARIMA model improves in the longer horizon. The Theil's U statistic also indicates that the state space local level model with deterministic seasonal and SARIMA model outperform the naïve model at most of the forecasting horizons. In conclusion, we recommend that the state space model should be used to forecast in the shorter and medium range forecasting horizons of the Nigeria's crude oil export. Howbeit, for longer forecasting horizon, the Box-Jenkins model should be considered. Furthermore, although not exploited in this present study, in practical applications, for a more accurate forecast, practitioners should also consider the combination and averaging of 
State Space Versus SARIMA Modeling Strategies

forecasts generated from both methods as advocated by Winkler and Makridakis (1983) and Makridakis and Winkler (1983).

\section{Acknowledgements}

The author expresses sincere appreciation to Dr. MrsBenedictaEwaen Asemota, Mr. and Mrs. Asemota IroghamaAndrew for their encouragements. Appreciation equally goes to the Nigerian National Petroleum Corporation for making the data readily available. The author sincerely appreciate the constructive comments of the anonymous referees.

\section{References}

1. Ayinde, K. and Abdulwahab, H. (2013). Modeling and Forecasting Nigerian Crude Oil Exportation: Seasonal Autoregressive Integrated Moving Average Approach, International Journal of Science and Research, Vol 2(12), 245 - 249

2. Bowman, K.O. and Shenton, L.R. (1975). Omnibus test contours for departures from normality based on and ,Biometrika, 62, 243-250. http://dx.doi.org/10.1093/biomet/62.2.243.

3. Box, G.E.P. and Jenkins, G.M. (1976). Time Series Analysis, Forecasting and Control, 2nd ed., San Francisco: Holden-Day.

4. Chatfield, C. (2000). Time-Series Forecasting, Chapman \& Hall/ CRC. http://dx.doi.org/10.1201/9781420036206

5. Commandeur, J.J.F. and Koopman, S.J. (2007). An Introduction to State Space Time Series Analysis, Oxford, Oxford University Press.

6. Doguwa, S. I and Alade, S.O (2015). On Time Series Modeling of Nigeria's External Reserves, CBN Journal of Applied Statistics, Vol. 6, No. 1(a), $1-28$.

7. Durbin, J. and Koopman, S. (2001). Time Series Analysis by State Space Methods, Oxford, Oxford University Press.

8. Etuk, E.H and Amadi, E.H (2013). Multiplicative SARIMA modelling of Nigerian Monthly Crude Oil Domestic Production, Journal of Applied Mathematics \& Bioinformatics, Vol. 3, No. 3, 103 - 112.

9. Hamilton, J.D. (1994). Time Series Analysis, Princeton University Press, New Jersey.

10. Harvey, A.C. (1989). Forecasting, Structural time series models and the Kalman filter, Cambridge University Press, New York

11. Harvey, A.C. and Koopman, S.J. (1992) Diagnostic Checking of Unobserved Components Time Series Models, Journal of Business and Economics Statistics, Vol. 10, 4, 377-389

12. Harvey, A.C. and Peters, S. (1990). Estimation Procedures for 
Structural Time Series Models, Journal of Forecasting, 9, 89-108. http://dx.doi.org/10.1002/for.3980090203

13. Hendry, D.F. and Clements, M.P. (2003). Economic Forecasting: Some Lessons from Recent Research, Economic Modelling, Vol. 20, 301 329.

http://dx.doi.org/10.1016/S0264-9993(02)00055-X

14. Lewis, C.D. (1982). Industrial and Business Forecasting Methods. Butterworths, London.

15. Lewis, A. S. and Overton, M. (2008). Nonsmooth optimization via BFGS.http://www.optimizationonline.org/DB_FILE/2008/12/2172.pdf

16. Lucas, R.E. (1976) Econometrics policy evaluation: a critique, Carnegie-Rochester Series on Public Policy, 1, 19-46. http://dx.doi.org/10.1016/S0167-2231(76)80003-6

17. Makridakis, S. and Winkler, R.L. (1983). Averages of Forecasts: Some Empirical Results, Management Science, Vol. 29, No.9, 987-996. http://dx.doi.org/10.1287/mnsc.29.9.987

18. Newbold, P. and Granger, C.W.J. (1974). Experience with Forecasting Univariate Time Series and the Combination of Forecasts, Journal of the Royal Statistical Society, Series A, Vol.137, 131-165. http://dx.doi.org/10.2307/2344546

19. Nigerian National Petroleum Corporation, Annual Statistical Bulletin (2009) Draft edition, page 12. <https://www.nnpcgroup.com/

20. Salau, M.O. (1998). Arima Modelling of Nigeria's Crude Oil Exports, AMSE, Modelling, Measurement \& Control, Vol. 18, No. 1, 1-20.

21. Slutsky, E. (1927). The Summation of Random Causes as the Source of Cyclic Processes, Econometrica, 5, 105-146. http://dx.doi.org/10.2307/1907241

22. Winkler, R.L. and Makridakis, S. (1983). The Combination of Forecasts, Journal of Royal Statistical Society, Series A, 146, Part 2, 150-157. http://dx.doi.org/10.2307/2982011

23. Witt, S.F., \& Witt, C.A. (1992). Modeling and forecasting demand in tourism. London: Academic Press.

24. Wold, H. (1938). A Study in the Analysis of Stationary Time Series, 1st ed., Uppsala: Almqvist and Wiksells.

25. Yule, G.U (1927). On a Method of Investigating Periodicities in Disturbed Series, with Special Reference to Wolfer's Sunspot Numbers, Philosophical Transactions of the Royal Society of London, Series A, Vol. 226,pp.267-298.http://dx.doi.org/10.1098/rsta.1927.0007 